\title{
THERAPEUTIC MONITORING OF MOOD STABILIZERS IN BIPOLAR DISORDER: LITHIUM AND ANTICONVULSANTS
}

\section{H. Ben Ammar ${ }^{1}$, S. Ben Fadhel ${ }^{1}$, A. Ben Hamadi ${ }^{1}$, A. Hakiri ${ }^{1}$, R. Ben Massoued ${ }^{1}$, Z. El Hechmi ${ }^{1}$. « F » Psychiatry Departement, Razi Hospital, Tunisia.}

\section{BACKGROUND AND AIMS}

The treatment of bipolar disorder is based on mood stabilizers. Efficacy of lithium is well documented in the literature, making it the gold standard treatment. However, its use declines with the advent of anticonvulsants. This raises the question about the reasons for the choice of prescription and monitoring of mood stabilizers in practice. The aims of this study were to determine the prophylactic lithium response in patients followed for bipolar disorder and compared to those of anticonvulsants and assess the mood stabilizers monitoring procedures in clinical practice.

\section{MATERIALS AND METHODS}

A retrospective study was conducted, over a period of six months from July to December 2017, with patients followed for bipolar disorder stabilized under the same mood stabilizer (lithium Li or anticonvulsant Ac) for at least one year. The participants were divided into two groups according to the mood stabilizing treatment. The two groups were compared according to socio-demographic, clinical and evolutionary profiles as well as the prophylactic response to treatment. For each type of mood stabilizer, the monitoring procedures were compared with the recommendations. The primary endpoint was the adequacy of the main biological monitoring parameters to recommendations.

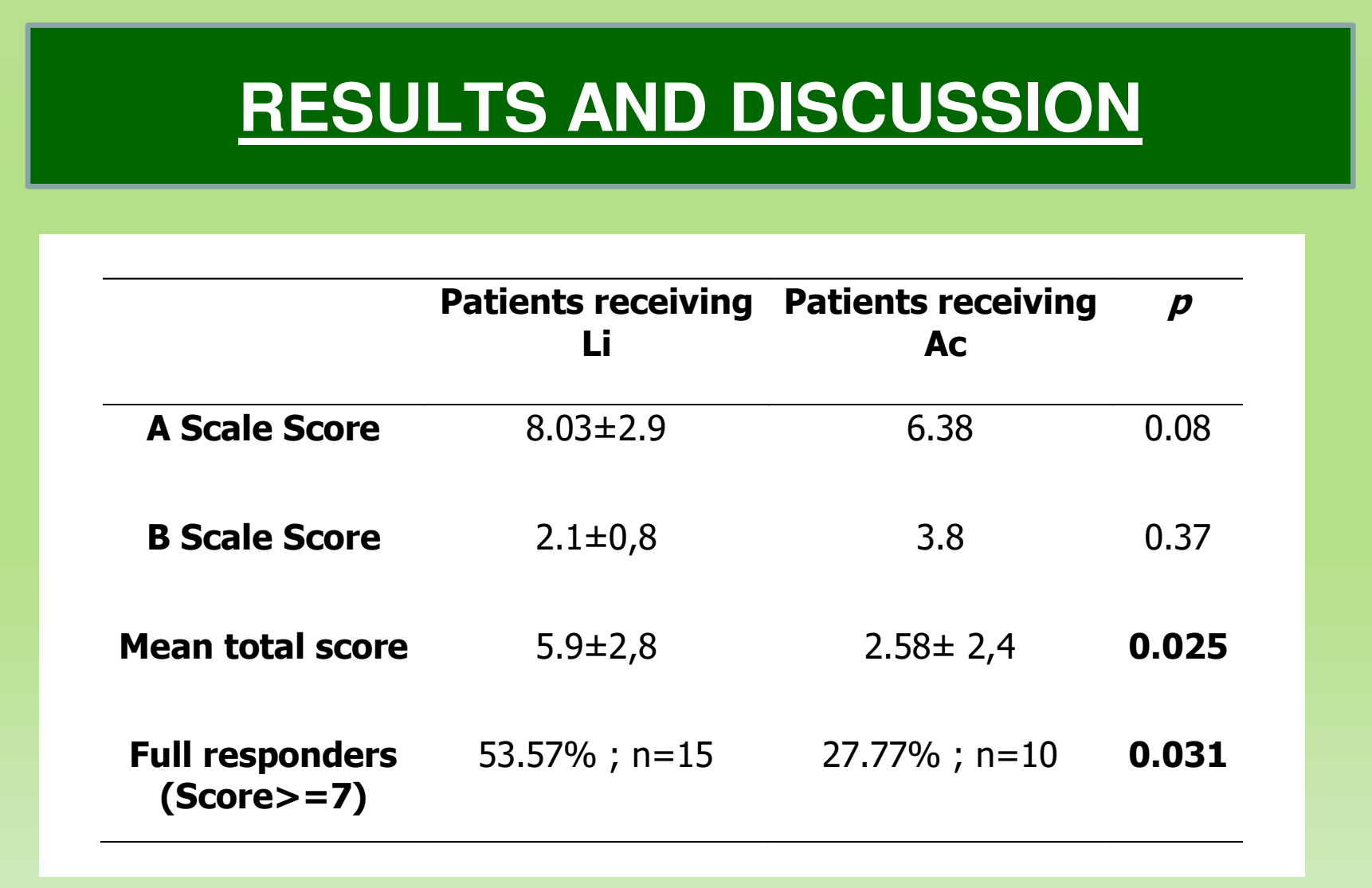

Comparison of the two groups of patients according to ALDA scale
Sixty-four patients were included in the study, 28 received lithium and 36 received anticonvulsants.

The socio-demographic profile and clinical characteristics were similar in two groups, except for the average total number of mood episodes, which was significantly lower in patients on lithium. Retrospective evaluation of the prophylactic response by ALDA scale showed a significantly higher mean total score in patients receiving lithium $(5.9 \pm 2.8$ versus $2.58 \pm 2.4$, $p$ $=0.025)$. $)$. Ten of them $(28.57 \%)$ were in compliance with the recommendations; while $19.44 \%$ of patients received anticonvulsants had all the monitoring parameters within the recommended time frame.

According to our results, the retrospective evaluation of the prophylactic response to the mood stabilizer treatment showed the superiority of Li over Ac, with a total response in $53.57 \%$ of patients under $\mathrm{Li}$. In the literature, the preventive efficacy of $\mathrm{Li}$ in bipolar disorder type I was estimated at 60 to $80 \%$ of good responders, with complete remission of the disorders; however, all types of bipolar disorder combined, this prophylactic response rate is only $50 \%{ }^{1}$. Indeed, naturalistic studies show that about one-third of patients do not respond to $\mathrm{Li}$ without being able to predict which patient will respond before two years of treatment ${ }^{1}$. In addition, a significant number of patients can not be maintained under $\mathrm{Li}$ because of intolerable side effects. This variability of the lithium response seems partly dependent on genetic factors.

\section{CONCLUSION}

Bipolar disorder is a severe disease that frequently requires therapeutic developments. Thymoregulators significantly modify the prognosis of this disease.

Practitioners will attach particular special attention to distinguish the therapeutic efficacy of the side effects of these drugs which are numerous and sometimes serious. A good knowledge of each of these products appears essential.

1. Burgess S, Geddes J, Hawton K, et al. Lithium for maintenance treatment of mood disorders. Cochrane Database Syst Rev 2002; 3: CD003013. 